\title{
Philosophiques
}

\section{Secrétariat, collaboration et auto-publication dans la France révolutionnaire}

\section{Sandrine Bergès}

Volume 44, numéro 2, automne 2017

Les nouveaux horizons du féminisme dans la philosophie francophone

URI : https://id.erudit.org/iderudit/1042333ar

DOI : https://doi.org/10.7202/1042333ar

Aller au sommaire du numéro

Éditeur(s)

Société de philosophie du Québec

ISSN

0316-2923 (imprimé)

1492-1391 (numérique)

Découvrir la revue

Citer cet article

Bergès, S. (2017). Secrétariat, collaboration et auto-publication dans la France révolutionnaire. Philosophiques, 44(2), 255-270.

https://doi.org/10.7202/1042333ar
Résumé de l'article

Comment publier ses écrits lorsqu'on est une femme française du dix-huitième siècle ? C'est une question que les femmes philosophes engagées de l'époque de la Révolution sont forcées de se poser : contribuer aux débats politiques aura peut-être un effet salutaire sur la place de la femme dans la société à venir.

Mais qui voudra dépenser de l'argent pour promulguer les écrits de celles qui ne pourront pas les défendre à l'Assemblée, puisqu'elles ne sont pas citoyennes?

Je proposerai trois modèles : celui de Madame Roland qui se présenta longtemps comme la compagne et secrétaire de son mari, et qui pourtant écrivit ses discours les plus importants ; celui de Sophie de Grouchy, qui collabora avec son mari Condorcet mais ne put publier ses propres écrits qu'après la Révolution ; enfin celui d’Olympe de Gouges, qui paya elle-même l'impression et la diffusion de ses nombreux écrits. Malgré leurs efforts, ces femmes furent remises à leur place plus ou moins violemment par une société révolutionnaire qui n'acceptait pas qu'elles puissent participer à ses réformes. 


\title{
Secrétariat, collaboration et auto-publication dans la France révolutionnaire
}

\author{
SANDRINE BERGÈS \\ Bilkent University \\ sandrineberges@gmail.com
}

\begin{abstract}
RÉSUMÉ. - Comment publier ses écrits lorsqu'on est une femme française du dix-huitième siècle? C'est une question que les femmes philosophes engagées de l'époque de la Révolution sont forcées de se poser: contribuer aux débats politiques aura peut-être un effet salutaire sur la place de la femme dans la société à venir.

Mais qui voudra dépenser de l'argent pour promulguer les écrits de celles qui ne pourront pas les défendre à l'Assemblée, puisqu'elles ne sont pas citoyennes?

Je proposerai trois modèles: celui de Madame Roland qui se présenta longtemps comme la compagne et secrétaire de son mari, et qui pourtant écrivit ses discours les plus importants; celui de Sophie de Grouchy, qui collabora avec son mari Condorcet mais ne put publier ses propres écrits qu'après la Révolution; enfin celui d'Olympe de Gouges, qui paya elle-même l'impression et la diffusion de ses nombreux écrits. Malgré leurs efforts, ces femmes furent remises à leur place plus ou moins violemment par une société révolutionnaire qui n'acceptait pas qu'elles puissent participer à ses réformes.
\end{abstract}

\begin{abstract}
What can a woman from $18^{\text {th }}$ Century in France do to get published? That is a question which women philosophers who were involved in politics in the revolutionary era were obliged to ask themselves. Contributing to political debates might have a beneficial effect on the status of women in the society of the future. But who would want to invest money to promulgate the work of those who could not defend it in the Assembly, because, as women, they were not citizens? I propose three models: that of Madame Roland, who represented herself for many years as the companion and secretary of her husband, and who nonetheless wrote his most important discourses; that of Sophie de Grouchy, who collaborated with her husband, Condorcet, but was only able to publish her own writing after the revolution; finally, that of Olympe de Gouges, who paid herself for the printing and distribution of her numerous works. Despite their efforts, these women were put in their place more or less violently by a revolutionary society that did not accept that they could participate in its reforms.
\end{abstract}

\section{Introduction}

Trop souvent, lorsqu'on parle des femmes dans l'histoire de la philosophie, et surtout de leur absence, on s'entend dire qu'avant le $\mathrm{xx}^{\mathrm{e}}$ siècle les femmes n'écrivaient pas de philosophie, qu'elles préféraient d'autres genres littéraires plus populaires, comme le roman ou l'histoire — avec le sous-entendu, bien sûr, que les femmes ne s'intéressaient pas aux choses abstraites. Remarquer cette absence des femmes de l'histoire de la philosophie n'est pas chose 
facile. Comme le remarque Michèle Le Dœuff dans L'étude et le rouet, on veut souvent nous faire croire que les temps ont changé et qu'il n'y a plus d'obstacles entre les femmes et la philosophie:

Si l'on considère le panthéon classique, la collection connue des «grands philosophes", on trouvera que c'est là une communauté masculine, et d'une manière que l'on peut même dire écrasante: complètement masculine, et de surcroit, franchement contente de l'être. De là à conclure que la rationalité philosophique est d'essence masculine, il n'y a qu'un pas, que certains et certaines franchissent. Au contraire, si l'on regarde le temps présent, on est en droit de croire qu'il n'y a plus de problème du tout. Rien n'interdit à une jeune femme d'entreprendre des études, puis des travaux philosophiques. Pourquoi, dès lors, ressasser une question caduque et parler d'avant-hier? [...] ne pourrait-on pas, maintenant, parler d'autre chose ? ${ }^{1}$

Geneviève Fraisse, dans les premières pages de La muse de la raison, nous renvoie au même problème: la question de l'intelligence et de l'âme de la femme n'est-elle pas, depuis le dix-neuvième siècle, «caduque » ? Et si elle l'est, pourquoi les philosophes devraient-ils en débattre?

Puisque la philosophie a refusé que la différence des sexes soit une de ses questions, et bien que ses textes ne soient pas si muets que l'on croit, l'histoire des textes, philosophiques et non philosophiques, pourrait nous aider à élaborer les termes les plus exacts possibles de la question ${ }^{2}$.

Or la question de la place des femmes dans la philosophie, presque trente ans après L'étude et le rouet et La muse de la raison, n'est certainement pas caduque. Cette place, si elle n'est pas disputée ouvertement, est cependant précaire. Les chiffres l'affirment: une étude nous montre que, même si les femmes forment la moitié des étudiants en philosophie de première année, ce pourcentage s'effrite petit à petit jusqu'à ce qu'il n'en reste que vingt pour cent aux derniers échelons de la profession ${ }^{3}$. La philosophie est toujours en grande partie un milieu masculin, et il n'est donc pas inutile de se pencher sur l'histoire de la discipline, afin d'étudier les techniques et procédés que nos ancêtres les femmes philosophes ont employés afin de lutter contre cette exclusion.

Ces dernières années, on a non seulement pu remarquer un effort visant à redécouvrir les femmes de l'histoire de la philosophie, mais aussi commencé à envisager que leur exclusion représente non pas une simple erreur de la part des historiens qui n'auraient pas remarqué leur existence,

1. Michèle Le Dœuff, L'étude et le ronet, Paris, Éditions du Seuil, 2008 [I989], p. I 5.

2. Geneviève Fraisse, La muse de la raison, Paris, Éditions Gallimard, I995, I9.

3. Molly Paxton, Carrie Figdor, Valerie Tiberius, "Quantifying the Gender Gap: An Empirical Study of the Underrepresentation of Women in Philosophy ", Hypatia 27 (4), 20I2, p. 949-957. 
mais bien une injustice épistémique, c'est-à-dire une injustice qui tient à l'exclusion d'un groupe d'individu dans un certain domaine d'expertise.

Dans Epistemic Injustice $(2007)^{4}$, Miranda Fricker soutient la thèse suivante: il existe un domaine où les vertus intellectuelles et les vertus morales (telles qu'elles sont définies par Aristote) se rejoignent, c'est-à-dire dans le regard que nous portons sur les personnes ou les groupes en tant que porteurs de connaissance. Fricker définit l'injustice testimoniale comme le manque de valeur que nous attribuons aux témoignages de certains individus. Il cite l'exemple de Tom Robinson dans le roman Ne tirez pas sur l'oiseau moqueur. Robinson, un homme noir du sud des États-Unis dans les années trente, est faussement accusé de viol. Mais puisqu'il est noir et que la victime est blanche, son témoignage est minoré. Cet exemple met en scène un témoignage au sens légal. Mais Fricker veut attirer notre attention sur les témoignages au sens figuré, c'est-à-dire sur toute transmission de connaissances $^{5}$. Cela comprend sans doute les écrits philosophiques, puisque ceuxci transmettent les efforts intellectuels d'un individu vis-à-vis de questions aussi profondes qu'essentielles à la communauté humaine présente et à venir. Lorsque les écrits d'un philosophe sont exclus des moyens que nous avons pour les transmettre et les partager, il s'agit donc d'une injustice testimoniale.

Cette exclusion opère à plusieurs niveaux:

Tout d'abord, un groupe peut être exclu des milieux d'apprentissage, formels (les écoles, universités) ou informels (les endroits où l'on parle). Cela a pour conséquence que les membres de ce groupe ont moins la possibilité d'accéder aux connaissances nécessaires pour devenir expert. Il ne s'agit pas ici d'exclure des philosophes ayant produit une œuvre, mais plutôt d'exercer un contrôle sur les individus qui pourraient le devenir. Ainsi, les portes des écoles, gardées jalousement par les philosophes, s'ouvrent seulement pour ceux qui sont reconnus comme membres du groupe dominant, et les autres ne pourront devenir philosophes à leur tour que par dissimulation ou en tant qu'exception. Comme le souligne Michèle Le Dœuff, cela n'est plus le cas absolument. Les femmes ne sont plus exclues des écoles ou des universités. Il reste cependant que le petit nombre de femmes philosophes du passé s'explique en partie par cet interdit.

Deuxièmement, les membres d'un groupe ayant accédé aux connaissances nécessaires par un moyen non traditionnel (les autodidactes) peuvent être exclus des groupes de recherche et des sociétés d'experts. Même si Fricker n'en parle pas, cela est le cas de bien des philosophes du passé. Margaret Cavendish, qui a acquis des connaissances scientifiques et philosophiques par ses lectures à l'âge adulte et passé des semaines entières à ne pas

4. Miranda Fricker, Epistemic Injustice: Power and the Ethics of Knowing, Oxford, Oxford University Press, 2007.

5. Miranda Fricker, Epistemic Injustice: Power and the Ethics of Knowing, p. 60, 6I, nI. 
quitter sa chambre, a su attirer l'attention de ses contemporains par sa notoriété, mais peu d'entre eux ont bien voulu dialoguer avec ses écrits.

Troisièmement, les personnes marginales auront sans doute plus de difficultés à disséminer leurs écrits, si toutefois elles parviennent à en produire dans de telles circonstances. Cavendish a pu imprimer ses livres parce que son mari en publiait d'autres qui se vendaient bien, et parce qu'il a bien voulu et su persuader son imprimeur de prendre ceux de sa femme à leur compte, bien sûr.

Enfin, même si leurs écrits sont publiés, ils ne seront peut-être pas lus, ou bien ils recevront des critiques négatives et condescendantes, dont la conséquence sera l'absence de réédition. Tel est encore le cas de Margaret Cavendish, dont les livres furent ridiculisés, puis oubliés, ce qui fait qu'il est aujourd'hui impossible de consulter une bonne partie d'entre eux.

Je ne vais pas insister ici sur ces manifestations de l'injustice épistémique liée à la représentation des femmes dans l'histoire de la philosophie. Je souhaite plutôt étudier la manière dont certaines femmes ont contourné ces obstacles afin de produire des écrits philosophiques. Je limiterai mon étude aux écrits en philosophie politique de trois femmes de la Révolution française: Olympe de Gouges, Marie-Jeanne Phlipon Roland, et Sophie de Grouchy. Cet article concerne donc la méthodologie de l'histoire de la philosophie, et de la philosophie elle-même, puisqu'il se penche sur les modes de travail philosophique de ces trois femmes. En même temps, il offre un aperçu de l'importance philosophique des arguments qu'elles ont pu, à travers ces méthodes, développer. Cependant, cela restera un aperçu, une invitation aux chercheurs en philosophie du dix-huitième siècle à se pencher sur les ouvrages de ces femmes.

\section{Les femmes philosophes de France durant la période révolutionnaire}

Comment publier ses écrits lorsqu'on est une femme française de la fin du dix-huitième siècle? C'est une question que les femmes philosophes engagées de l'époque de la Révolution sont forcées de se poser: contribuer aux débats politiques aura peut-être un effet salutaire sur la place de la femme dans la société à venir.

Mais qui voudra dépenser de l'argent pour promouvoir les écrits de celles qui ne pourront pas les défendre elles-mêmes à l'Assemblée, puisqu'elles ne sont pas citoyennes? Qui voudra s'exposer au ridicule en imprimant les écrits de celles qu'on ne saurait prendre au sérieux quand elles parlent de politique?

Pendant une brève période, les femmes ont pu espérer que cela allait changer, qu'elles deviendraient citoyennes au même titre que les hommes. Cet espoir motiva certains écrits, comme ceux d'Eta Palm d'Aelders et bien sûr ceux de Mary Wollstonecraft en Angleterre ${ }^{6}$.

6. Eta Palm D'Aelders présenta son projet d'inclusion des femmes en politique en juillet I79I. La même année, Mary Wollstonecraft dédiait son essai sur les droits de la femme à Talleyrand, afin que celui-ci pense à inclure les femmes dans sa réforme de l'éducation nationale. 
Malheureusement, la Terreur interrompit ce mouvement et entraîna une répression violente des femmes qui souhaitaient s'engager politiquement, dont la mort de deux de nos protagonistes, Olympe de Gouges et Manon Roland, qui furent guillotinées à l'automne I793. Quelques jours avant leur mort, le journal jacobin Le Moniteur avait publié un article réprimandant les femmes qui pensaient avoir une place dans la Révolution, les avertissant qu'une bonne citoyenne devait se borner à élever les citoyens de l'avenir et non tenter d'influencer le cours du gouvernement ${ }^{7}$.

Mais même pendant la première période, plutôt encourageante, du début de la Révolution, la place d'une femme intellectuelle active n'était pas gagnée d'avance, les femmes n'étant pas acceptées dans les sociétés savantes ou les académies. Nous devons noter ici l'exception qui fut faite pour Louise Keralio-Robert et Marie Le Masson Le Golft, toutes deux élues membres honoraires de l'Académie d'Arras sous présentation par Robespierre - fait qui poussa Alyssa Sepinwall à qualifier Robespierre de féministe de l'Ancien Régime $^{8}$. Il est pourtant à noter que Louise Keralio n'était pas féministe et qu'elle soutenait que les femmes ne pouvaient ni ne devaient prendre une part active au gouvernement ${ }^{9}$. Marie Le Masson Le Golft, dont l'expertise touchait aux sciences naturelles et non pas aux sciences politiques, avait des tendances plutôt traditionalistes et, dès $\mathrm{I} 789$, se mit à l'abri des excès révolutionnaires en se contentant de donner des cours privés ${ }^{10}$.

On conclut donc que les femmes de la période révolutionnaire restaient pour la plupart exclues de la politique et du gouvernement, et qu'on ne recherchait pas dans leurs écrits des conseils politiques. Elles furent donc forcées d'utiliser des méthodes d'écriture qui leur permettraient de percer indirectement l'obscurité où elles se trouvaient cantonnées. Malheureusement, ces méthodes non traditionnelles qui n'entrent pas dans le registre des "écrits philosophiques» ont eu pour effet leur oubli. En posant qu'il va de notre intérêt en tant que femmes philosophes d'aller rechercher l'œuvre de ces femmes par-delà leur obscurité, je présente ci-dessous une introduction ayant pour but de faciliter cette recherche.

7. Charles-Aimé Dauban, Étude sur Madame Roland et son temps, Paris, Henri Plon, I864, p. ccxlix.

8. Alyssa Sepinwall, «Robespierre, Old Regime Feminist? Gender, the Late Eighteenth Century, and the French Revolution Revisited ", The Journal of Modern History, 82, 2010, p. I-29.

9. Annie Geffroy, "Louise de Keralio-Robert, pionnière du républicanisme sexiste», Annales historiques de la Révolution française, 344, 2006, p. I07-I24, I I0.

10. Olivier Perru, "Marie Le Masson Le Golft (I749-I826). Le progrès des idées là où on ne l'attend pas ", Journal of Interdisciplinary History of Ideas, 2006, 5 (9), article 2. 


\section{La secrétaire et auteure secrète: Marie-Jeanne Roland}

Marie-Jeanne Roland (Madame ou Manon Roland), née Phlipon, se présenta longtemps comme la compagne et secrétaire de son mari, alors que c'est pourtant elle qui écrivit ses discours les plus importants.

Née à Paris d'une famille de la bourgeoisie artisane en I748, MarieJeanne bénéficia d'une éducation minimale mais libre - c'est-à-dire que ses parents ne l'empêchaient pas de lire ce qui lui tombait sous la main. Ainsi, elle découvrit Plutarque à huit ans et fut dès lors convertie au républicanisme. Dès son plus jeune âge, elle remplissait des cahiers de ses essais philosophiques et politiques, et à vingt ans elle participa au concours de l'Académie de Besançon portant sur l'énoncé "Comment l'éducation des femmes pourrait contribuer à rendre les hommes meilleurs» (I 777$)^{11}$. Roland défend dans ce discours une théorie républicaine de la place des femmes dans la nation, inspirée du modèle de Sparte. Les femmes, selon elle, ne doivent pas participer au gouvernement, mais comme la république repose sur le bien-être des familles et que la femme est en grande partie responsable de l'administration du foyer, il s'ensuit qu'elles doivent être libres et non sous la domination de leurs maris, telles des esclaves. Par ailleurs, elle soutient, marquant ses distances avec le modèle antique qu'elle admire pourtant, qu'une république qui tolère l'esclavage n'en est pas une.

L'essai pour l'Académie de Besançon fut soumis anonymement. Il ne reçut pas de prix et ne fut donc pas publié. Parmi ses autres écrits non publiés, on trouve: plusieurs discours philosophiques dont un sur la liberté, un mémoire sur la grossesse et la maternité, des centaines de lettres offrant des réflexions sur la philosophie, sur la politique (avant et pendant la Révolution), sur l'esthétique, et, bien sûr, ses fameuses Mémoires et notices historiques qui furent publiées après sa mort. Parmi ses écrits publiés durant sa vie, qui le furent tous anonymement, on trouve plusieurs lettres politiques publiées par son ami Brissot dans le Patriote français, et un journal de voyage en Angleterre.

La vie et l'œuvre de Madame Roland démontrent à la fois une forte réticence à se présenter comme auteur et un grand désir d'influencer le cours de l'histoire par ses écrits. Une de ses dernières réflexions avant sa mort fut qu'elle aurait voulu devenir la Catherine Macaulay de son pays ${ }^{12}$. Mais avant la Révolution, elle écrivait que les femmes ne devaient pas signer leurs œuvres par égard pour leur réputation:

Jamais je n'ai eu la plus légère tentation de devenir auteur un jour; je vis de très bonne heure qu'une femme qui gagnait ce titre perdait beaucoup plus qu'elle n'avait acquis. Les hommes ne l'aiment point, et son sexe la critique: si

11. L. A. Champagneux, Euvres de J. M. Ph. Roland, femme de l'ex-ministre de l'intérieur, tome troisième, Paris, Bidault, I799-I 800 .

12. Claude Perroud, Mémoires de Madame Roland, nouvelle édition critique, tome II, Paris, Plon, I905, p. 264. 
ses ouvrages sont mauvais, on se moque d'elle, et l'on fait bien; s'ils sont bons, on lui les ôte. Si l'on est forcé de reconnaitre qu'elle en a produit la meilleur partie, on épluche tellement son caractère, ses mœurs, sa conduite et ses talens, que l'on balance la réputation de son esprit par l'éclat que l'on donne à ses défauts ${ }^{13}$.

Cet interdit ne l'empêcha cependant pas de publier, que ce soit de façon anonyme pour le Patriote français de Brissot au début de la Révolution ou bien sous le nom de son mari tout au long de leur mariage:

Durant douze années de ma vie, j'ai travaillé avec mon mari, comme j’y mangeais, parce que l'un m'était aussi naturel que l'autre. Si l'on citait un morceau de ses ouvrages, où l'on trouvât plus de grâces de style; si l'on accueillait une bagatelle académique dont il se plaisait à envoyer le tribut aux sociétés savantes dont il était membre, je jouissais de sa satisfaction, sans remarquer plus particulièrement si c'était ce que j'avais fait; et il finissait par se persuader que véritablement il avait été dans une bonne veine, lorsqu'il avait écrit tel passage qui sortait de ma plume. Au ministère, s'il s'agissait d'exprimer des vérités grandes ou fortes, j'y mettais toute mon âme; il était tout simple que son expression valut mieux que les efforts d'un secrétaire. J'aimais mon pays; j'étais enthousiaste de la liberté: je ne connaissais point d'intérêts ni de passions qui pussent entrer en balance avec eux ${ }^{14}$.

Roland était une grande lectrice de Rousseau et attirée par l'idéal féminin qu'il proposait. Ce choix même n'est pas sans intérêt philosophique : Roland retient le républicanisme rural de Rousseau et l'importance qu'il accorde à la vie familiale. Elle en retire une théorie toute particulière du bien commun et de la vertu civique. Ce sont les femmes qui, en tant que mères, non seulement sont la source du bonheur de la nation, mais le définissent. C'est-à-dire que les législateurs, selon Roland, devraient se renseigner dans les foyers avant de décider des besoins de la nation. Les familles, et donc les mères, forment les racines de la République.

Même si elle accroît par cette théorie le pouvoir et l'influence des femmes dans la nation, la combinaison des tendances républicaines et rousseauistes de Roland résulte en une position conflictuelle, avec d'un côté le sens de son devoir de citoyenne philosophe et de l'autre celui de sa position de femme, donc de pilier du foyer. En tant que citoyenne, elle se doit de déployer ses qualités de philosophe. En tant que femme, elle doit les refouler pour prendre en charge les besoins de son mari et de sa fille.

Cependant, il semble qu'à la fin de sa vie Roland ait changé d'avis et qu'elle ait accepté que le rôle de chacun doive être défini par rapport à son

13. Saint-Albin Berville, et Jean-François Barrière, Mémoires de Madame Roland, avec une notice sur sa vie, des notes et des éclaircissements historiques, $3 \mathrm{e}$ édition, Paris, Baudoin Frères, I 827, p. I99-200.

14. Saint-Albin Berville, et Jean-François Barrière, Mémoires de Madame Roland, avec une notice sur sa vie, des notes et des éclaircissements historiques, p. 200-20I. 
habileté, et non par rapport à son sexe. Ainsi, dit-elle, les femmes de tous les temps, si elles en sont capables, feraient mieux de travailler sur des "projets de lettres ou de circulaires ou d'affiches» que de passer leur temps en intrigues ${ }^{15}$.

Mais avant son emprisonnement, la résolution (peu satisfaisante) de ce conflit est rendue possible par la méthode qu'elle a choisie, l'anonymat, ainsi qu'avec l'aide de la carrière politique et académique de son mari. Ce n'est que lorsqu'elle est sur le point de perdre la vie, et alors qu'elle vient d'abandonner son mari (par choix, puisqu'elle est amoureuse d'un autre) et sa fille et son foyer (par contrainte, puisqu'elle est en prison) qu'elle change d'avis et décide que ses Mémoires seront publiées à son nom.

Puisque par souci d'anonymat elle a choisi de ne pas se présenter comme philosophe, nous ne l'avons pas reçue comme telle, et nous avons perdu une variation sur le thème des valeurs républicaines qui vient placer la famille, le foyer et la femme au fondement du développement de ces valeurs.

\section{La collaboratrice anonyme: Sophie de Grouchy}

Sophie de Grouchy est connue pour son traité philosophique sur la sympathie, qu'elle écrivit en I 792 et publia en I 798 en épilogue de sa traduction de la Théorie des sentiments moraux d'Adam Smith ${ }^{16}$. La traduction ellemême ne manque pas de valeur philosophique. Le texte de Smith avait fait l'objet de deux traductions qui étaient davantage des résumés et commentaires truffés d'inexactitudes et de confusions. La traduction de Grouchy, au contraire, est de qualité: elle fut rééditée en I982 et en 2016 , et il n'existe qu'une autre édition de qualité comparable ${ }^{17}$. D'après son biographe Guillois, elle publia cette traduction à un moment où elle avait besoin d'argent - pour elle-même, sa fille et sa sœur — puisque ses avoirs ainsi que ceux de Condorcet avaient été confisqués par l'État quand ce dernier avait été proscrit.

Les lettres sur la sympathie sont (avec la traduction de Smith) le seul texte certain qu'il nous reste de Sophie de Grouchy. Pourtant, nous savons par son mari, Condorcet, qu'elle en écrivit d'autres. Dans son testament à sa fille, rédigé quelques semaines avant sa mort, il recommande à Éliza, en vue de son éducation morale et philosophique, de lire ses propres écrits et les Lettres sur la sympathie de Sophie, et ajoute que "d'autres fragments de sa mère donneront sur le même sujet des vues très utiles ${ }^{18}$ ".

15. Saint-Albin Berville, et Jean-François Barrière, Mémoires de Madame Roland, avec une notice sur sa vie, des notes et des éclaircissements historiques, p. 203.

16. Adam Smith et Sophie de Grouchy Condorcet, Théorie des sentiments moraux, Paris, F. Buisson, I798.

17. Adam Smith, Théorie des sentiments moraux, traduit et annoté par Michaël Biziou, Claude Gautier et Jean François Pradeau, Paris, Presses Universitaires de France, I999.

18. J.-A.-N. Condorcet, A. O'Connor et F. Arago, Euvres de Condorcet, tome premier, Paris, Firmin Didot Frères, I849, p. 624. 
On peut par ailleurs attester que les Lettres sur la sympathie étaient déjà rédigées à ce moment. Ce sont elles que Condorcet recommande à sa fille dans son Testament au tout début de 1794, et Grouchy en parle dans sa correspondance avec Étienne Dumont au printemps 92 :

Voici les manuscrits informes dont j'ai parlé à M. Dumont, ou plutôt les indéchiffrables brouillons. J'ai égaré la $8^{\mathrm{e}}$ lettre sur la sympathie. Quand à l'autre fatras, il n'y a encore que quelques faibles traces de développement de passion et de caractère, et ils ne sont encore appuyés sur aucune des circonstances dont le roman peut tirer son plus grand intérêt. Une des grandes causes de ma paresse pour continuer c'est: $\mathrm{I}^{*}$ la difficulté d'avoir un bon conseil (m'en arrivera-t-il d'outremer!) 2* la crainte de manquer de moyens d'exécution relativement aux idées dont un pareil sujet en d'autres mains pourrait être enrichi, et qui pâlira probablement entre les miennes.

Si M. Dumont voulait venir diner ici mardi, je serais plus à portée de profiter de ses conseils, mais aussi peut-être seront-ils plus francs par écrit. Je n'ai pas besoin de lui demander de ne parler a personne de ce que je lui envoie ${ }^{19}$.

On retiendra plusieurs points intéressants de cette lettre. D'une part, il semble que Sophie de Grouchy avait déjà rédigé les huit lettres en I792. D'autre part, elle écrivait un roman - se peut-il que ce «fatras" soit les fragments utiles dont parle Condorcet, ou bien Grouchy avait-elle aussi commencé un autre travail en philosophie morale? En tant qu'aristocrate et salonnière, elle était en mesure de solliciter des commentaires de la part d'amis écrivains - Dumont avait rédigé les discours de Mirabeau, et à l'époque où Grouchy lui écrit, il traduisait l'œuvre de Bentham en français. Nous n'avons pas la réponse de Dumont à Grouchy — mais il semble probable que cet échange se soit déroulé autour du dîner proposé, donc, malheureusement pour nous, à l'oral.

Dans les Lettres sur la sympathie, Grouchy ne se borne pas à commenter les théories de Smith, mais développe également les siennes, non seulement sur l'origine de la sympathie (qu'elle attribue aux tous premiers contacts d'un bébé avec sa nourrice), mais aussi sur sa fonction politique. Selon elle, les relations de domination sont seulement possibles lorsqu'il n'existe pas de sympathie entre les citoyens, c'est-à-dire lorsqu'ils sont incapables de se concevoir les uns les autres comme capable des mêmes émotions, aussi bien plaisirs que douleurs. Ses arguments touchent aussi à l'éducation: la sympathie morale nécessite, dit-elle, réflexion et abstraction. Pour ce faire, il est nécessaire que les enfants reçoivent une éducation leur permettant de développer leurs capacités intellectuelles.

19. Jean Martin, Achille du Chastellet et le premier mouvement républicain en France d'après des lettres inédites (1791-1792) Paris, L. Maretheux, Imprimeur de la Cour d'Appel, I927, La Révolution française (I93 I) 34, p. 105-32, I2I. 
Puisqu'on sait que Grouchy écrivait et qu'il ne nous reste pourtant qu'un ouvrage de petite taille signé par elle, il faut envisager la possibilité qu'elle ait publié certains de ses écrits sous le couvert de l'anonymat. J'ai défendu ailleurs l'hypothèse suivante: Grouchy collabora avec son mari Condorcet à des textes politiques publiés dans Le Républicain ${ }^{20}$. Ce journal (dont il ne parut que quatre numéros) publia des articles de Thomas Paine, d'Achille Duchâtelet, et de Condorcet lui-même. Pourtant, seuls Paine et Duchâtelet signent leurs articles (et Duchâtelet signe aussi celui de Paine!) ${ }^{21}$. On peut donc supposer que, parmi les articles anonymes, il y en a deux qui sont issus de la plume de Grouchy. Le style de ces articles - La « Lettre d'un jeune mécanicien ", les «Observations sur le mémoire du Roi » — de même que sa correspondance avec Étienne Dumont de $\mathrm{I} 792$ le suggèrent ${ }^{22}$.

Une autre collaboration jusqu'ici méconnue pourrait être liée à l'écriture et à la publication du dernier ouvrage de Condorcet, l'Esquisse d'un tableau des progrès de l'esprit humain ${ }^{23}$. Cet ouvrage recèle en effet des thèmes et arguments qui sont communs aux écrits des deux auteurs, sur la liberté comme absence de domination, sur le rôle de la sympathie dans le développement de la moralité, et sur la justice et l'éducation. De plus, la version publiée par Grouchy diffère du manuscrit de Condorcet en ce qu'elle propose une théorie plus féministe de l'histoire, encadrant les théories de Condorcet dans une présentation de la famille, et donc aussi des femmes comme agents du progrès:

L'habitude d'une vie plus sédentaire avait établi entre les deux sexes une plus grande égalité. Les femmes ne furent plus considérées comme un simple objet d'utilité, comme des esclaves seulement plus rapprochées du maitre. L'homme y vit des compagnes, et apprit enfin ce qu'elles pouvaient pour son bonheur. Cependant, même dans les pays où elles furent le plus respectées, où la polygamie fut proscrite, ni la raison ni la justice n'allèrent jusqu'à une entière réciprocité dans les devoirs ou dans le droit de se séparer, jusqu'à l'égalité dans les peines portées contre l'infidélité. [...]

L'histoire de cette classe de préjugés et de leur influence sur le sort de l'espèce humaine, doit entrer dans le tableau que je me suis proposé de tracer; et rien

20. Sandrine Bergès, «Sophie de Grouchy on the Cost of Domination in the Letters on Sympathy and Two Anonymous Articles in Le Républicain» The Monist 98 (I), 201 5, p. IO2II 2 .

21. Étienne Dumont, Sonvenirs sur Mirabean et sur les deux premières assemblées législatives, Paris, Librairie de Charles Gosselin, I832, p. 333.

22. "Il faut mettre dans les annales de notre liberté le signet à la page du retour du roi. » Jean Martin, Achille du Chastellet et le premier mouvement républicain en France d'après des lettres inédites (1791-1792), p. I05-32, I I3.

23. Sandrine Bergès, "Sophie de Grouchy and the Publication of Condorcet's Sketch of Human Progress: a Tale of Exclusion ", à paraître dans le Journal of the History of Ideas. 
ne servira mieux à montrer jusqu'à quel point son bonheur est attaché aux progrès de la raison ${ }^{24}$.

Cet ajout a été ignoré en raison du fait que l'édition de Grouchy a été remplacée (parce qu'elle ne correspondait pas exactement au manuscrit) par celle de Louis Arago au dix-neuvième siècle. Ainsi, cet angle féministe d'un ouvrage qui eut beaucoup d'influence a disparu, et nous n'en avons pas bénéficié.

\section{Aventures dans l'auto-publication: Olympe de Gouges}

Olympe de Gouges est sans doute la plus connue des trois femmes qui font l'objet de cet article. C'est peut-être, paradoxalement, parce qu'elle est celle qui bénéficia le moins du support d'un mari (elle devint veuve très jeune). Gouges travailla seule toute sa vie, et finança elle-même l'impression et la diffusion de ses nombreux écrits. Ce que l'on connaît d'elle est surtout sa Déclaration des Droits de la Femme et de la Citoyenne. Mais elle écrivit aussi de nombreuses pièces de théâtre, chacune précédée d'une introduction prenant la forme d'un traité philosophique sur l'esclavage, le mariage et autres thèmes; des placards, diffusés dans Paris; des discours où elle proposait des réformes dès le début de la Révolution, se battant sans cesse contre l'injustice en société et au sein des familles; et finalement un discours philosophique, Le bonheur primitif de l'homme ou rêveries patriotiques. On ne parle jamais de ce dernier, surtout pas dans les milieux philosophiques. C'est l'envers, peut-être, de notre jugement, pourtant bien fondé, suivant lequel les femmes philosophes du passé utilisaient souvent des genres non traditionnels, plus littéraires que philosophiques, pour produire leurs arguments. On ne s'attend donc pas à un traité philosophique de la part d'une femme ayant produit des pièces de théâtre.

Les pièces de théâtre, surtout leurs préfaces, et les "placards " portent en grande partie sur l'injustice sociale. Gouges était reconnue par ses contemporains comme ayant introduit des arguments contre l'esclavage dans la France révolutionnaire avant même que Brissot n'ait eu l'idée de fonder sa Société des Amis des Noirs ${ }^{25}$. C'est d'ailleurs grâce à lui que la pièce de Gouges sur ce sujet, Zamore et Mirza, fut finalement jouée au Théâtre français, cinq ans après qu'elle y eut été reçue.

L'argument de Gouges est à la fois simple et original. Les esclaves, ditelle, sont différents des maîtres uniquement par rapport à l'éducation qu'ils ont reçue : c'est-à-dire que, sans éducation, tous les humains seraient égaux. Mais à l'encontre de ce à quoi on pourrait s'attendre, et de ce que défend par

24. J.-A-N. Condorcet et Sophie de Grouchy, Esquisse d'un tablean historique des progrès de l'esprit humain. Ouvrage posthume de Condorcet, Paris, Agasse, rue des Poitevins, $\mathrm{n}^{\circ}$ I 8. L'an III de la République, une et indivisible [I795], p. 47-48.

25. Olivier Blanc, Marie-Olympe de Gouges: 1748-1793: des droits de la femme à la guillotine, Paris, Tallandier, 20I4, p. 89. 
exemple Mary Wollstonecraft en parlant des femmes comme des esclaves, Gouges ne conclut pas que la solution dépende de l'éducation des esclaves ${ }^{26}$. Au contraire, il semble qu'elle attribue le blâme non à l'ignorance des esclaves, mais plutôt aux connaissances des maîtres, qui, dit-elle, leur font croire qu'ils sont, tels des dieux, élevés au-dessus de la nature.

Cette méfiance par rapport à l'instruction se retrouve aussi dans son traité sur le bonheur primitif, où Gouges défend le point de vue de Rousseau dans son premier discours, où il soutient que les arts et les sciences sont en général néfastes au bonheur de l'humanité. Mais Gouges ne fait pas que suivre Rousseau : ce dernier, dit-elle, prend l'humanité dans son état primitif pour animale, c'est-à-dire pour des êtres incapables de moralité, de réflexion ou de véritable bonheur humain. Gouges argumente que Rousseau a tort et que si les êtres primitifs manquent d'instruction, ils ne manquent pourtant pas de la sorte d'intelligence qui permet de former des amitiés, des relations de confiance et des familles. Gouges entreprend de distinguer deux formes de développement intellectuel: l'instruction, qui est d'après elle souvent néfaste parce qu'elle nous amène à nous croire supérieurs aux autres, et l'émulation, qui tient de la nature et nous rend à même de développer nos vrais caractères et nos vraies forces dans des contextes naturels où ils seront utiles. L'émulation était un concept fort controversé à la fin du dix-huitième siècle. Bernardin de Saint Pierre n'y voyait qu'une compétition dangereuse, issue de la jalousie ${ }^{27}$. Mais d'autres, comme l'abbé Roubaud ou Marmontel, s'inspiraient de Cicéron et pensaient l'émulation comme imitation de la vertu, c'est-à-dire comme la mimesis d'Aristote ${ }^{28}$. C'est sans doute de ces derniers que se rapprochait Gouges et il est à noter que, lorsqu'à la fin du traité elle propose un programme d'éducation sociale, elle le fait par le théâtre, proposant que les enfants des pauvres reçoivent une formation d'acteurs, et que les représentations théâtrales, au lieu de distractions pour les riches, servent à éduquer le peuple ${ }^{29}$. Cet esprit pédagogique du théâtre est sans doute hérité de son père naturel, lui-même auteur de pièces, qui était proche du fils de Racine et écrivit avec lui sur les réflexions de son père à propos de la Poétique d'Aristote ${ }^{30}$.

26. Mary Wollstonecraft, A Vindication of the Rights of Men, A Vindication of the Rights of Woman, Oxford, Oxford University Press, I993, p. I02.

27. Jacques Henri Bernardin de Saint Pierre, Les vœux d'un solitaire, Paris, L'imprimerie de Monsieur, I789, p. I99, 204.

28. Voir abbé Roubaud, Nouveaux synonymes françois, tome second, Liège, Plomteux, I786, p. 85-89; Jean-François de Marmontel, Bélisaire, nouvelle édition, I 823, Londres, S. Low, p. 99. Cicéron définit l'émulation comme l'imitation de la vertu dans les Tusculanes, IV, I7.

29. Olympe de Gouges, Le bonheur primitif de l'homme, ou les rêveries patriotiques, Amsterdam, Royer, I789, p. 75-78.

30. Le père présumé d'Olympe de Gouges, Jean-Jacques Le Franc de Pompignan, luimême tragédien, était proche de Louis Racine et publia une Lettre sur le théâtre en general et sur les tragédies de Jean Racine en particulier (I752). 
Les textes d'Olympe de Gouges sont sans aucun doute riches en arguments philosophiques. Mais ils ne sont que difficilement accessibles: il n'existe pas d'édition moderne du Bonheur primitif, et on est obligé pour le lire de se pencher sur des éditions du dix-huitième siècle ${ }^{31}$. Pourtant ces textes présentent un contraste intéressant, non seulement avec les vues de Rousseau, mais aussi avec les discussions philosophiques sur l'éducation de la même période, notamment de Mary Wollstonecraft.

\section{Leçons républicaines}

La pensée républicaine n'est pas toujours vue en tant que pensée féministe. Plusieurs femmes philosophes ont critiqué cette pensée en raison de ses racines aristotéliciennes et de son insistance sur la vie publique ${ }^{32}$. On peut penser, en particulier, que le citoyen républicain a besoin, pour participer pleinement à la politique de sa nation, d'une femme à la maison qui puisse s'occuper de ses enfants et de son foyer. Pour cette raison, les femmes philosophes, sauf certaines exceptions notables, n'ont pas fait l'objet du renouveau d'intérêt pour la pensée républicaine - que ce soit de la part de ceux qui firent l'histoire de cette pensée (nous pensons ici à Quentin Skinner), ou bien de ceux portés par un intérêt plus contemporain envers la pensée républicaine (nous pensons cette fois-ci à Philip Pettit) ${ }^{33}$. En ce qui concerne l'aspect historique des études républicaines, il est pourtant vrai que les femmes de la fin du XviII ${ }^{e}$ siècle, en Angleterre comme en France, ont défendu des idées centrales au républicanisme. Pour Wollstonecraft, comme pour Macaulay, la liberté est l'absence de domination. Cela signifie, entre autres, que l'on n'est pas libre si on est sous l'emprise d'une autre personne, même si cette personne ne choisit pas d'exercer son pouvoir sur nous; c'està-dire, tant qu'il sera possible à quelqu'un d'autre que nous-mêmes de mettre fin à nos projets sans nous en donner raison. Pour Grouchy, Roland et Gouges, ce pouvoir arbitraire qui domine peut être celui d'un roi, d'un mari, ou bien des lois et institutions. Toutes étaient philosophes républicaines, et ne pas l'admettre non seulement aurait pour conséquence de passer outre de nombreux arguments qui ont pour souche des idées républicaines, mais encore nous empêcherait de découvrir un nombre de solutions (parfois embryonnaires) aux problèmes que les idées républicaines constituent, eu égard au féminisme. J'ai présenté un aperçu de ces arguments dans les sections précédentes, et il reste maintenant à les joindre les uns aux autres

31. On peut les retrouver sur Gallica, le moteur de recherche de la Bibliothèque nationale de France: [http://gallica.bnf.fr/ark:/I 2 I 48/bpt6k42599j.r=Le+bonheur+primitif + de $+1 \%$ $\mathrm{E}_{2} \% 80 \%$ 99homme, +ou+les+r \% C3\% AAveries+patriotiques.langFR]

32. Voir par exemple Anne Phillips, «Feminism and Republicanism: Is This a Plausible Alliance?" Journal of Political Philosophy, 8 (2), 2000, p. 279-293; Carole Pateman, "Why Republicanism ? ", Basic Income Studies, 2 (2), 2007, p. I-6.

33. La philosophe Cécile Laborde est une exception notable, Critical Republicanism: The Hijab Controversy and Political Philosophy, Oxford, Oxford University Press, 2008. 
afin de montrer l'importance de la pensée féminine républicaine pour ne plus qu'elle soit effacée des dialogues et études philosophiques, contemporains et à venir.

Nous retrouvons certains thèmes et réflexions chez les trois auteurs dont j'ai parlé: en particulier, chacune d'entre elles étudie la famille, les relations humaines, et l'éducation, trois sujets qui se rapprochent davantage du foyer que de la politique, et qui pourtant, pour ces auteurs, sont au centre du bon développement de la société républicaine. Chacune reconnaît l'importance du foyer pour la république. Peut-être est-ce dû à l'influence de Rousseau, qu'elles ont en commun, et en particulier à sa préférence pour un républicanisme rural, c'est-à-dire un État soutenu par des familles et des villages qui se consacrent à produire ce dont ils ont besoin, et vivent sainement, loin de l'attrait malsain des citadelles ${ }^{34}$. Dans ce modèle, le travail de la femme au sein du foyer, l'éducation des enfants, la formation des idées républicaines et le soutien émotionnel et physique de l'unité familiale sont au cœur du bon fonctionnement de la république. C'est l'idéal de Manon Roland, mais aussi celui de De Gouges, qui, dans son Bonheur primitif, présente un tableau de sociétés heureuses, saines, et où l'inégalité et la domination n'ont pas de prise. Les femmes y sont égales, mais leur rôle reproductif et éducatif est estimé à sa juste valeur.

Grouchy partage, semble-t-il, cet idéal des familles républicaines dans la mesure où elle est convaincue que le développement moral des citoyens commence à la naissance, à travers les relations d'un nouveau-né avec sa nourrice. C'est à ce moment-là que les sensations qui portent plus tard à la sympathie prennent naissance. Il faut noter, par ailleurs, qu'à l'encontre de Rousseau qui idéalise la relation de la mère avec son nouveau-né en insistant sur l'allaitement, et également de Wollstonecraft, qui écrit qu'une mère qui n'allaite pas ses enfants ne mérite pas d'être citoyenne, Grouchy propose seulement la présence d'une nourrice dans la vie du nouveau-né ${ }^{35}$. Qu'elle soit la mère de l'enfant ou bien une employée de la famille, cela n'aura pas de conséquences puisque c'est un effet purement physiologique qui doit se produire. En effet, l'enfant doit apprendre à reconnaître le plaisir et la douleur, et créer un lien physique de dépendance avec une personne afin de pouvoir reconnaitre sa douleur et de pouvoir en souffrir.

Puisque la sympathie, qui pour Grouchy entraîne la moralité, débute par les sensations, elle nécessite une certaine éducation. Il s'agit de savoir reconnaître la douleur et le plaisir des autres, et en même temps d'être

34. Voir Jean-Jacques Rousseau, Projet de constitution pour la Corse [I763] (édition préparée par Jean-Marie Tremblay pour "Les classiques des sciences sociales»), Université du Québec à Chicoutimi, bibliothèque Paul-Emile-Boulet.

35. Voir Jean-Jacques Rousseau, Émile ou De l'éducation, Paris, Garnier Flammarion, I966, p. 37; Mary Wollstonecraft, The Vindication of the Rights of Woman, Oxford, Oxford University Press, I994, p. 227; Sophie de Grouchy, Lettres sur la sympathie (édition préparée par Bernier et Dawson), Voltaire Foundation, Oxford University Press, 2010, p. 4 I. 
capable d'abstraction afin de comprendre que certaines douleurs sont morales plutôt que physiques. Tout citoyen, conclut Grouchy, doit donc recevoir une éducation en partie dans les matières abstraites de raisonnement afin de pouvoir traiter les autres citoyens en égaux. Sans cela, remarquet-elle, il n'est pas possible pour un riche et un pauvre de se mesurer l'un à l'autre et de se reconnaître comme appartenant à la même espèce. Ils n'auront donc aucune sympathie l'un pour l'autre ${ }^{36}$.

Roland, comme Gouges, ont aussi reconnu l'importance de l'éducation pour le bien-être des citoyens républicains, mais davantage que Grouchy, elles ont voulu suivre le programme de Rousseau, c'est-à-dire, l'éducation par la nature. À cet égard, nous avons vu le scepticisme de Gouges vis-à-vis de ce qu'elle appelle l'instruction, c'est-à-dire l'enseignement de faits, de textes à la mode, et d'autres choses que l'enfant ne comprend pas mais qui lui donne une notion mal fondée de sa propre supériorité. Au contraire, écrit Gouges, " [on] ne devrait instruire les jeunes gens que quand ils commencent à développer leurs connaissances, leurs goûts, et leurs penchants ${ }^{37}{ }^{3}$. C'està-dire que, comme le propose Rousseau, on doit laisser la nature pousser l'enfant à choisir par lui-même ce qu'il veut savoir, et quand il veut le savoir. Bien sûr, en ce qui concerne Rousseau, cela s'applique uniquement aux enfants de sexe masculin, mais Gouges, comme Roland, ne relève pas ce problème, choisissant plutôt de voir dans l'Émile un programme d'éducation universelle et égalitaire. Cela contrairement à Wollstonecraft, qui s'appliqua à dénoncer le sexisme du système d'éducation proposé par Rousseau ${ }^{38}$. Gouges et Roland - cette dernière dériva de l'Émile un programme d'éducation pour sa fille - ont au contraire choisi de ne pas retenir le Rousseau qui pense les femmes comme inférieures. Lorsque Gouges parle du théâtre et de son rôle dans un état républicain, elle évite toute référence à la Lettre à D’Alembert, texte qui présente un mélange d'arguments contre le théâtre et de fulminations contre les femmes.

Le manque de prise de position et la position universelle asexuée constituent souvent un obstacle à une critique féministe de positions philosophiques. Si l'on ne s'attarde pas à la place donnée aux femmes dans un système, on ne peut espérer en résoudre les problèmes. Mais cette objection ne s'applique pas aux femmes républicaines de la fin du dix-huitième siècle. Leur position de départ est toujours celle d'une femme, et elles puisent à chaque instant dans leur vie de femme afin de proposer des observations justes sur des sujets qui leur semblent décisifs pour le succès de la pensée républicaine. On ne peut donc pas les accuser d'oublier qu'elles sont femmes,

36. Sophie de Grouchy, Lettres sur la sympathie, p. IоI.

37. Olympe de Gouges, Le bonheur primitif de l'homme, ou les rêveries patriotiques, p. 56 .

38. Mary Wollstonecraft, The Vindication of the Rights of Woman. 
et que certains philosophes refusent de voir en elles des citoyennes à part entière.

\section{Conclusion: vers une pratique plus inclusive?}

Ce qui est vrai pour Olympe de Gouges est aussi le cas pour les trois femmes philosophes qui constituent l'objet de cet article. Pour les découvrir, il s'agit de se livrer à un travail de détective, ou tout du moins de sortir de certaines habitudes du travail académique. Les femmes du dix-huitième siècle ne pouvaient toujours pas écrire à la manière des hommes. Elles devaient déguiser leurs efforts, parfois se les cacher à elles-mêmes, comme Madame Roland qui ne croyait pas en l'égalité des sexes, et souvent sacrifier leur propre gloire à la postérité, comme Grouchy, qui a préféré collaborer avec son mari et n'a publié sous son propre nom qu'après la mort de celui-ci, quand elle avait besoin d'argent. Ou encore, comme Olympe de Gouges, risquer le ridicule, et puis plus tard tomber dans l'oubli.

Lorsque les femmes philosophes de la période révolutionnaire sont invisibilisées, non seulement nous perdons une preuve de l'existence des femmes philosophes dans l'histoire, mais aussi nous passons sous silence une perspective féminine importante sur la pensée républicaine. Cette perspective est presque entièrement absente des réappropriations actuelles de la pensée républicaine, ce qui entraîne le scepticisme des philosophes féministes par rapport à cette philosophie politique. On peut espérer qu'en relisant les femmes philosophes du dix-huitième siècle nous pourrons avancer vers une pensée républicaine plus inclusive.

Une approche plus inclusive de l'histoire de la philosophie devra donc retracer le chemin qui a jeté ces femmes dans l'obscurité, découvrir leurs écrits philosophiques au milieu de textes et de contextes qui ne le sont pas toujours, et rajouter leur nom quand elles ne l'ont pas signé. Les bénéfices sont évidents: en se penchant sur les ouvrages oubliés de ces femmes, nous accédons à des arguments de l'époque révolutionnaire, souvent républicains et féministes, qui tiennent compte de la place du foyer et des femmes dans la vie politique. 